\title{
Non-monotonic density dependence of the diffusion of DNA fragments in low-salt suspensions
}

\author{
Mathieu G. McPhie ${ }^{1}$ and Gerhard Nägele ${ }^{1, *}$ \\ ${ }^{1}$ Institut für Festkörperforschung, Forschungszentrum Jülich, D-52425 Jülich, Germany
}

\begin{abstract}
The high linear charge density of 20-base-pair oligomers of DNA is shown to lead to a striking nonmonotonic dependence of the long-time self-diffusion on the concentration of the DNA in low-salt conditions. This generic non-monotonic behavior results from both the strong coupling between the electrostatic and solvent-mediated hydrodynamic interactions, and from the renormalization of these electrostatic interactions at large separations, and specifically from the dominance of the far-field hydrodynamic interactions caused by the strong repulsion between the DNA fragments.
\end{abstract}

With the increasing importance of biophysics there is a greater overlap between the subjects of the physics of biological molecules and colloidal physics, which deals with small particles in suspension. Many of the techniques that have been developed in colloidal physics are directly applicable to biological molecules, such as proteins, and cells. DNA in particular is a very interesting biomolecule which exhibits a wide range of behaviors, due to its interactions with proteins and enzymes, but also due to its physical characteristics. A particular feature of DNA that is of interest to colloidal scientists is its high linear charge density $[1,2]$. The interaction energy between DNA molecules and the structure of suspensions of DNA fragments have been shown to be strongly affected by this charge density. Consequently, other effects associated with a strong charge, such as electrolyte screening, electrolyte friction, charge condensation, charge inversion and like-charge attraction in the presence of multivalent salt ions, have also been predicted and investigated [3-6]. Several investigations have considered the effect on the structure due to the renormalization of charge $[2,7]$. Little work, however, has been performed on the interesting consequences of a high charge on the dynamics of these molecules.

Highly charged colloids present an entirely different paradigm of particle interactions to the classical hardsphere model. Well-known features of a low-salt suspension of charged colloids are the low osmotic compressibility of the suspension, and that the mean distance between the particles scales with the inverse of the cube root of the colloid concentration over a wide range of concentrations [8]. This scaling corresponding to the formation of a correlation hole around the particles, leads to the domination of the far-field over the near-field hydrodynamic interactions (HI), and therefore to an altered dynamic behavior which is unlike that of hard-sphere colloids.

Wilk et al. [1] have measured the long-time translational self-diffusion coefficient, $D^{L}=\left(D_{\|}^{L}+2 D_{\perp}^{L}\right) / 3$, of isotropic dispersions of 20 base pair oligomers of DNA by fluorescence correlation spectroscopy for various salt concentrations. DNA is a suitable molecule for studying effects resulting from electrostatic and hydrodynamic coupling due to its large linear charge density of approx- imately $-2 e / 3.4 \AA$. The 20 -mer DNA are almost perfectly monodisperse, rigid cylindrical rods with length $L=6.8 \mathrm{~nm}$ and diameter $d=2 \mathrm{~nm}$ (aspect ratio of 3.4), a bare valency of $Z=-42$ in neutral or basic $p \mathrm{H}$ solutions and the translational free diffusion coefficient of $D^{0}=1.07 \times 10^{-6} \mathrm{~cm}^{2} \mathrm{~s}^{-1}$. The long-time coefficient, $D^{L}$, was found to have an unexpected non-monotonic concentration dependence in low-salt conditions.

In this letter we describe a versatile theoretical scheme that we have developed for the calculation of $D^{L}$ in colloidal systems $[3,9,10]$. This scheme includes the long-range far-field part of the HI between the particles, which dominates in low-salt suspensions. We will show that in combination with colloid charge renormalization, this scheme successfully describes the non-monotonic dependence of $D^{L}(\phi)$ on the macroion volume fraction $\phi$. This effect is of general importance and may be observed in any dispersion of colloids or biomolecules where long-range repulsive interactions are prevalent. The nonmonotonicity in $\phi$ is unusual since it requires a delicate interplay of $\mathrm{HI}$ and electrosteric repulsions over a sufficiently broad concentration range. In contrast, a nonmonotonic $\phi$-dependence is not uncommon for transport properties such as the primary electroviscous coefficient, $p(\phi)$, associated with the suspension viscosity $\eta[11]$, and the collective diffusion coefficient $D^{c}$ [8]. Moreover, the electrophoretic mobility $\mu[12]$ as well as $1 / \eta$ and $D^{L}(\phi=0)$ exhibit a minimum as a function of the electrolyte concentration. The minimum in $D^{L}(0)$ is found for globular macroions $[9,13]$, and also for semi-flexible charged polymers as shown recently in experiments and simulations [14]. In all cases considered, HI play a decisive role, e.g., the maximum of $D^{c}(\phi)$ arises from a balance of the slowing HI and the speed-up of density relaxations caused by the electrosteric repulsion. The maximum in $p(\phi)$ at intermediate salinity arises from a competition between the velocity gradient inside the macroion double layer that grows with $\phi$, and the shrinking double layer distortion [11]. Ignoring HI can lead to nonphysical results such as the failure to predict the maximum in the electrophoretic mobility of a short polyelectrolyte chain as a function of the monomer number $[15,16]$.

Due to the increasing power of computers, substan- 
tial progress has been made in the simulation of transport properties in non-dilute charged colloidal dispersions. Formerly, simulations that include the electrokinetic effect of electrolyte ions have been difficult due to the large asymmetry between these components, both in size and charge. These simulations have focused largely on the challenging problem of electrophoresis [12, 17, 18], frequently used for particle characterization but for which a complete theory in dense systems, in which there is strong overlap of the electrical double layers, is still lacking. Our theoretical scheme is therefore also presented as a significant step in developing a versatile statistical mechanical tool to describe the electrokinetic transport in non-dilute suspensions.

Our scheme is based on an exact memory equation for the self-intermediate scattering function of colloids undergoing overdamped Brownian motion. The irreducible memory function in this equation is approximated using the idealized mode-coupling theory for Brownian fluids, with the important extension of including the HI between all ionic species. Instead of solving the mode-coupling equations fully self-consistently, which would be a very challenging numerical task in the presence of HI, we use a simplified solution scheme which retains analytical simplicity and yields only small differences in the numerical results [19].

According to our scheme, the long-time coefficient $D^{L}$ of a non-dilute suspension is given by the StokesEinstein-like relation [3]

$$
\frac{D^{L}}{D^{0}}=\left[1+\frac{\Delta \zeta^{C F}}{\zeta^{0}}+\frac{\Delta \zeta^{E F}}{\zeta^{0}}\right]^{-1},
$$

which includes, in addition to the colloid-solvent friction $\zeta^{0}=6 \pi \eta^{0} a$, where $\eta^{0}$ is the solvent viscosity and $a$ the colloid radius, a colloid friction (CF) and an electrolyte friction $(\mathrm{EF})$. The colloid friction arises from the microion-averaged electrosteric and HI between the colloids, and is present even when the microion degrees of freedom are ignored. It is given in our scheme by [3]

$$
\frac{\Delta \zeta^{C F}}{\zeta^{0}}=\frac{n}{6 \pi^{2}} \int_{0}^{\infty} d k k^{2} \frac{\left[h(k)-\frac{1}{D^{0}} h^{d}(k)\right]^{2}}{2+n\left[h(k)+\frac{1}{D^{0}} h^{d}(k)\right]},
$$

where $n$ is the colloid number density, $h(k)$ is the total correlation function of the colloids, $h^{d}(k)$ is the distinct hydrodynamic function of the colloids, and $D^{0}$ is the freediffusion constant. This expression for $\Delta \zeta^{C F}$ only requires the colloid static structure factor $S(k)=1+n h(k)$. Eq. (2) is the zeroth order term in the expansion of the total long-time friction coefficient, $\Delta \zeta=\Delta \zeta^{C F}+\Delta \zeta^{E F}$, in terms of the colloid-microion mobility ratio $D^{0} / D_{i}^{0}$. Eq. (1) states that the extra friction due to the fast kinetics of the mobile salt ions, $\Delta \zeta^{E F}$, is given by the difference of the total friction coefficient and $\Delta \zeta^{C F}$ [3]. The $\mathrm{EF}$ is due to the non-instantaneous relaxation of the mi- croionic atmosphere. We have derived a simple expression for the long-time EF contribution, valid for the case when the free diffusion coefficients of the various salt ion species, $D_{i}^{0}$, are much greater than the colloid free diffusion coefficient. This expression is

$$
\begin{aligned}
& \frac{\Delta \zeta^{E F}}{\zeta^{0}}=\frac{2}{3 \pi^{2}} \sum_{i=1}^{m} n_{i} \frac{D^{0}}{D^{0}+D_{i}^{0}} \int_{0}^{\infty} d k k^{2} \times \\
& \left\{\frac{[1+n h(k)] \frac{1}{D^{0}} h_{c i}^{d}(k)-h_{c i}(k)\left[1+n \frac{1}{D^{0}} h^{d}(k)\right]}{2+n\left[h(k)+\frac{1}{D^{0}} h^{d}(k)\right]}\right\}^{2},
\end{aligned}
$$

where the sum goes over all microion species of number density $n_{i}$, and where $h_{c i}(k)$ and $h_{c i}^{d}(k)$ are the partial total correlation and partial distinct hydrodynamic functions between the microions and the colloids. The EF contribution to $D^{L}$ is significant in very dilute systems but is negligible when the mobility difference between the colloidal spheres and the microions is large, and when $\phi$ is increased. The second finding is attributed to the enhanced homogenization of the electrolyte background with increasing $\phi[3]$.

Since the dynamic effect of the microions is small, we can simplify the problem by considering the colloids as interacting with an effective pair potential. In the resulting one-component model of weakly charged colloids, the effective interaction between colloids of radius $a$ and bare valency $Z_{\text {bare }}$, follows the repulsive part of the DerjaguinLandau-Verwey-Overbeek (DLVO) potential [20],

$$
\beta u(r)=L_{B} Z_{\text {bare }}^{2}\left(\frac{e^{\kappa a}}{1+\kappa a}\right)^{2} \frac{e^{-\kappa r}}{r}, \quad r>2 a
$$

where $L_{B}=e^{2} /\left(\epsilon k_{B} T\right)$ is the Bjerrum length in a solvent of dielectric constant $\epsilon$ and $\kappa$ is the inverse Debye screening length determined by the concentration of added salt ions and monovalent counterions. In a 1:1 electrolyte solution, $\kappa^{2}=4 \pi L_{B}\left(2 n_{s}+n\left|Z_{\text {bare }}\right|\right)$. For strongly charged colloids where $L_{B}\left|Z_{\text {bare }}\right| / a>1$, this potential is still suitable but only with the charge and screening parameter replaced by an effective charge, $Z_{\text {eff }}$, and screening parameter, $\kappa_{\text {eff }}$, due to the condensation of counterions near the colloid surfaces. There exist several schemes for the calculation of these effective quantities, and these have recently been of considerable interest. Those mostly used are the cell model approximation of Alexander et al. [21], and the renormalized jellium approximation (RJA) [22].

The RJA for the effective macroion charge in a closed suspension with a fixed salt concentration, as opposed to a system in contact with a reservoir, involves numerically solving the Poisson equation for the mean electric potential $\psi(r)$ of a single colloidal sphere, surrounded by a Boltzmann-distributed microion cloud and a uniform negatively-charged background of charge density, $n Z_{\text {eff }}$, that describes the jellium representing the other macroions. The resulting equation for the reduced po- 
tential, $y(r)=\beta e \psi(r)$, is

$$
\nabla^{2} y(r)=4 \pi L_{B}\left[2 n_{s} \sinh (y(r))+n Z_{\text {eff }}\left(e^{y(r)}-1\right)\right]
$$

with the boundary conditions $y(\infty)=0, y^{\prime}(\infty)=$ 0 , and $y^{\prime}(a)=-L_{B} Z_{\text {bare }} / a^{2}$. The numerical solution of Eq. (3) is asymptotically matched to the solution of the linearized equation, $\nabla^{2} y_{\text {lin }}(r)=\kappa_{\text {eff }}^{2} y_{\text {lin }}(r)$, where $\kappa_{\text {eff }}$ is the effective screening parameter $\kappa_{\text {eff }}^{2}=$ $4 \pi L_{B}\left(2 n_{s}+n\left|Z_{\text {eff }}\right|\right)$. The effective charge comes from the linearized solution at the inner boundary, $y_{\text {lin }}^{\prime}(a)=$ $-L_{B} Z_{\text {eff }} / a^{2}$. Since the effective charge also appears in Eq. (3) this solution procedure must be iterated until self-consistency in $Z_{\text {eff }}$ has been established [22].

With the so-determined interaction potential, $S(k)$ is calculated using standard integral equation theories. For this study, we use the rescaled mean spherical approximation (RMSA) [23], known to be a reasonably accurate theory of the structure of charged colloids.

Eq. (2) has been developed for spherically symmetric particles and is therefore not directly applicable to rodlike particles in high-salt suspensions. On the other hand, in the low-salt suspensions, as measured by Wilk et al., the microstructure is mostly determined by the long-range electrostatic monopole term which is spherically symmetric. Therefore, we treat the DNA fragments as spheres with an effective radius, $a_{\text {eff. This ra- }}$ dius appears in the solution of the effective charge and thus in the calculated structure factor. The largest effect of $a_{\text {eff }}$ however, is to determine the scaling used to map the volume fraction dependent calculations onto the weight concentration dependent measurements by $c(\mathrm{~g} / \mathrm{L})=3 \phi M_{w} /\left(4 \pi a_{\text {eff }}^{3} 10^{3} N_{A}\right)$, where $M_{w}=13022 \mathrm{~g}$ $\mathrm{mol}^{-1}$ is the molecular weight of the DNA fragments. The effective radius resulting from the Stokes-Einstein relation applied to the measured diffusion coefficient at infinite dilution is $a_{\mathrm{eff}}=2.0 \mathrm{~nm}$. However, since the excluded volume interaction of the effective spheres is influential at high salinity only, from comparing our results with the high-salt measurements of Wilk et al., we have determined $a_{\text {eff }}=3.4 \mathrm{~nm}=L / 2$ to provide the best overall fit, independent of the salt concentration. An effective radius of half the molecular length has some parallels with the excluded volume calculations of rod-like particles in the isotropic state. Since the bare charge is given by the number of ionizable groups on the DNA molecule, $a_{\text {eff }}=L / 2$ is the only adjusted parameter.

The effective charge for our model of the DNA fragments, calculated via the RJA in a closed system as in the experiment, is presented in Fig. 1. For low-salt systems, $Z_{\text {eff }}$ shows a non-monotonic dependence on $\phi$. At very low $\phi$, there is also a non-monotonic dependence of $Z_{\text {eff }}$ on the salt concentration (c.f., Fig. 1), with the limit that $Z_{\text {eff }} \rightarrow Z_{\text {bare }}$ when $n \rightarrow 0$ and $n_{s} \rightarrow 0$. This zero- $\phi$ non-monotonicity of $Z_{\text {eff }}$ is also seen in its expansion in terms of the bare charge $Z_{\text {bare. }}$ If $Z_{\text {bare }}$ is sufficiently

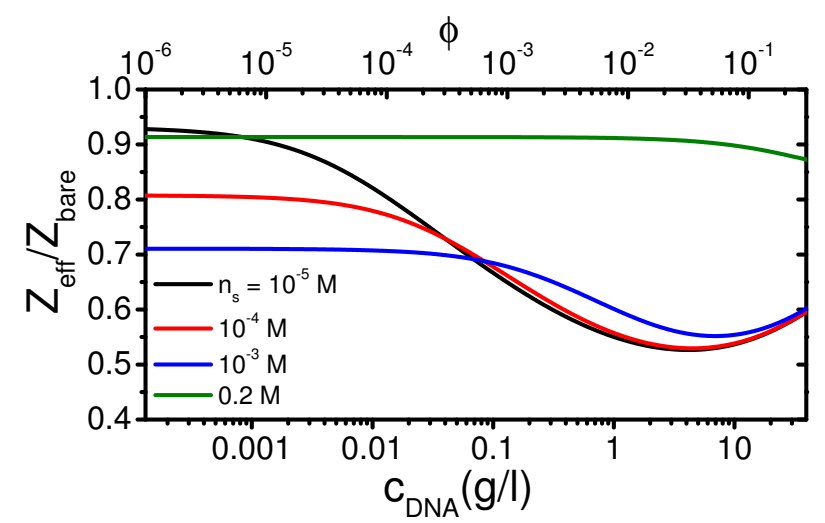

FIG. 1: Effective charge of 20 -mer DNA $\left(Z_{\text {bare }}=-42\right)$, determined via the RJA, as a function of DNA and salt concentrations.

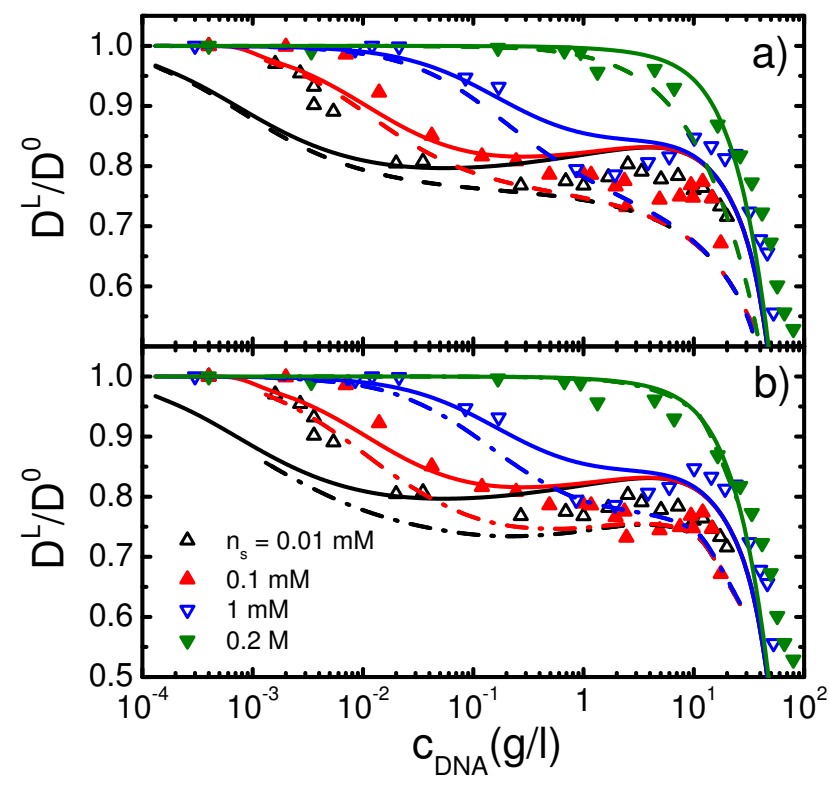

FIG. 2: Self-diffusion coefficient $D^{L}$ of the DNA model vs weight concentration for salt concentrations as indicated. Symbols are experimental results taken from [1]. a) Comparison with theoretical results using $Z_{\text {eff. Solid lines are results }}$ with HI, dashed lines are without HI. b) Comparison with theoretical results with HI. Solid lines are again results using $Z_{\text {eff }}$, dashed lines are results with fixed charge $Z=-42$.

large, $Z_{\text {eff }}$ becomes independent of the bare charge. This is the so-called saturated effective charge, $Z_{\text {eff }}^{\text {sat }}$. In our system, $\left|Z_{\text {bare }} L_{B} / a_{\mathrm{eff}}\right| \approx 9$ which is well into the nonlinear regime, but $Z_{\text {eff }}$ is less than $66 \%$ of $Z_{\mathrm{eff}}^{\mathrm{sat}}$, so that saturation is not yet reached. Even though the $\kappa_{\text {eff }}$ depends on $Z_{\text {eff }}$, it shows no non-monotonic $\phi$-dependence.

The measured $D^{L}$ of the 20-mer DNA and the comparison with the results of our spherical model are shown in Fig. 2. In Fig. 2a, we include the results of our scheme when far-field HI are included (solid lines) or ig- 
nored (dashed lines), with the values $Z_{\text {eff }}$ and $\kappa_{\text {eff }}$ used in both cases. The non-monotonic dependence of $D^{L}$ on $\phi$ in the experiments is also seen in the theoretical predictions when HI between the particles are included. If the $\mathrm{HI}$ are ignored, $D^{L}$ shows a monotonic $\phi$-dependence, for all considered salt concentrations. In Fig. 2b, a comparison is made between the theoretical results for $D^{L}$, including the effects of $\mathrm{HI}$, where instead of a $\phi$-dependent $Z_{\text {eff }}$ (solid lines again) we use a fixed charge of $Z=-42$ (dash-dotted lines). For both sets of results a non-monotonic $\phi$-dependence is seen. The non-monotonicity, however, is much stronger when the non-constant $Z_{\text {eff }}$ is used. This suggests that the non-monotonic $\phi$-dependence of the experimental $D^{L}$ results from a simultaneous interplay between the hydrodynamic enhancement caused by far-field HI, and the nonmonotonic $Z_{\text {eff }}$ in low-salt systems. The strong decline in $D^{L}$ at large $\phi$ seen in Fig. 2 is due to electrosteric caging which becomes stronger with increasing $\phi$. The singlemacroion EF effect described in Booth's theory [13] can not explain the non-monotonicity of $D^{L}$ since it is significant only for $\phi<10^{-4}$ and $n_{s} \approx 0.01 \mathrm{M}$ [3], which is a salt concentration much larger than those where the DNA- $D^{L}$ behaves non-monotonically.

The hydrodynamic enhancement of the diffusion of particles repelling each other over long distances results from the fact that the dominant far-field HI advect neighboring particles that may otherwise have hindered the motion of the considered one. Near-field HI, on the other hand, have the opposite effect of slowing the diffusive motion. Hydrodynamic enhancement of $D^{L}$ has been seen before in suspensions of charged and magnetically interacting colloidal particles [19, 24], and in simulations with HI of charged nano-sized polyions [25], but without a visible non-monotonic $\phi$-dependence. A nonmonotonic $D^{L}(\phi)$ was found in simulations of salt-free polyelectrolyte solutions [26]. In these simulations, however, HI have been neglected and the values for $D^{L} / D^{0}$ are smaller than 0.1 , which is the value where the freezing transition of charged spheres and rods occurs [27].

In summary, we have shown that the non-monotonic concentration dependence of $D^{L}$ in low-salt suspensions of DNA fragments can be understood by the influence of far-field $\mathrm{HI}$ and charge renormalization. According to our scheme, the non-monotonicity of $D^{L}$ is a generic effect for any low-salt suspension of strongly charged small colloids or bio-molecules. We have obtained this result using a simplified mode-coupling scheme. This scheme is a marked improvement on previous methods, since its many-component version includes the far-field HI between all ionic species. Its analytic simplicity allows the study of electrokinetic phenomena such as the EF effect on self- and collective diffusion for non-zero concentrations.

This work and the printing of the article was under appropriation of funds from the Deutsche Forschungsgemeinschaft (SFB-TR6, TP B2). We acknowledge A. Banchio, J. Gapinski and A. Patkowski for discussions.

Electronic address: g.naegele@fz-juelich.de

[1] A. Wilk, J. Gapinski, A. Patkowski, and R. Pecora, J. Chem. Phys. 121, 10794 (2004).

[2] X. Qiu, L. W. Kwok, H. Y. Park, J. S. Lamb, K. Andresen, and L. Pollack, Phys. Rev. Lett. 96, 138101 (2006).

[3] M. G. McPhie and G. Nägele, J. Chem. Phys. 127, 034906 (2007).

[4] S. Pianegonda, M. C. Barbosa, and Y. Levin, Europhys. Lett. 71, 831 (2005).

[5] X. Qiu, K. Andresen, L. W. Kwok, J. S. Lamb, H. Y. Park, and L. Pollack, Phys. Rev. Lett. 99, 038104 (2007).

[6] E. Allahyarov, G. Gompper, and H. Löwen, Phys. Rev. E 69, 041904 (2004).

[7] L. F. Rojas-Ochoa, R. Castañeda-Priego, V. Lobaskin, A. Stradner, F. Scheffold, and P. Schurtenberger, Phys. Rev. Lett. 100, 178304 (2008).

[8] A. J. Banchio and G. Nägele, J. Chem. Phys. 128, 104903 (2008).

[9] M. Kollmann and G. Nägele, J. Chem. Phys. 113, 7672 (2000).

[10] M. G. McPhie and G. Nägele, J. Chem. Phys.: Condens. Matter 16, S4021 (2004).

[11] E. Ruiz-Reina, P. Garcia-Sanchez, and F. Carrique, J. Phys. Chem. B 109, 5289 (2005).

[12] V. Lobaskin, B. Dünweg, M. Medebach, T. Palberg, and C. Holm, Phys. Rev. Lett. 98, 176105 (2007).

[13] U. Geigenmüller, Chem. Phys. Lett. 110, 666 (1984).

[14] X. Schlagberger, J. Bayer, J. O. Rädler, and R. R. Netz, Europhys. Lett. 76, 346 (2006).

[15] K. Grass, U. Böhme, U. Scheler, H. Cottet, and C. Holm, Phys. Rev. Lett 100, 096104 (2008).

[16] S. Frank and R.G. Winkler, Europhys. Lett. 83, 38004 (2008).

[17] T. Araki and H. Tanaka, Europhys. Lett. 82, 18004 (2008).

[18] K. Kim, Y. Nakayama, and R. Yamamoto, Phys. Rev. Lett. 96, 208302 (2006).

[19] G. Nägele and P. Baur, Physica A 245, 297 (1997).

[20] E. J. M. Verwey and J. T. G. Overbeek, Theory of the Stability of Lyophobic Colloids (Elsevier, Amsterdam, 1948).

[21] S. Alexander, P. M. Chaikin, P. Grant, G. J. Morales, and P. Pincus, J. Chem. Phys. 80, 5776 (1984).

[22] S. Pianegonda, E. Trizac, and Y. Levin, J. Chem. Phys. 126, 014702 (2007).

[23] J.-P. Hansen and J. B. Hayter, Mol. Phys. 46, 747 (1982).

[24] K. Zahn, J. M. Méndez-Alcaraz, and G. Maret, Phys. Rev. Lett. 79, 175 (1997).

[25] M. Jardat and P. Turq, Z. Phys. Chem. 218, 699 (2004).

[26] R. Chang and A. Yethiraj, J. Chem. Phys. 116, 5284 (2002).

[27] H. Löwen, T. Palberg, and R.G. Simon, Phys. Rev. Lett. 70, 1557 (1993). 